http://jmscr.igmpublication.org/home/ ISSN (e)-2347-176x ISSN (p) 2455-0450

crossref DOI: https://dx.doi.org/10.18535/jmscr/v8i1.116

\title{
Tuberculosis Presenting as Migratory Polyarthralgia in an Immunocompetent Patient: A Case Report
}

\author{
Authors \\ Himanshu Dhiman*, Rajeev Kumar, Joban Preet Singh Deol \\ Vardhman Mahavir Medical College and Safdarjung Hospital, New Delhi
}

\begin{abstract}
We present an atypical case of tuberculosis in an immunocompetent man from New Delhi, India. The patient entered the hospital with pain in left hip for two months. He had a history of joint pains involving both small and large joints for past two months, which was migratory in nature. There were no constitutional symptoms of fever, night sweats, weight loss or loss of appetite. Diagnosis of tuberculosis was made by full body FDG-PETCT Scan, followed by histopathology of the biopsy from the enlarged supraclavicular lymph node. Tuberculosis cases with such presentation are rather known with immunodeficient patients. Extrapulmonary tuberculosis, especially skeletal tuberculosis is seen more frequent in young immunocompetent patients. The migratory nature of the joint pains is rather unknown in cases of skeletal tuberculosis and needs more evaluation of such cases in the future.
\end{abstract}

\section{Introduction}

Worldwide, TB is one of the major causes of death and the leading cause from a single infectious agent (above HIV/AIDS). The incidence and prevalence of this disease are directly related to the degree of poverty and eradication requires prevention, early diagnosis and institutional support. TB with pulmonary involvement is the most common form of presentation, and in extrapulmonary sites, lymph nodes are the most common, followed by pleural, osteoarticular, military, genitourinary, peritoneal and central nervous system involvement. Pulmonary TB can spread by contiguity, hematogenous dissemination or by swallowing infected lung secretions. TB presents with symptoms like fever, night sweats, cough (in pulmonary involvement) which may be absent at disease onset and may be productive or not, anorexia and weight loss. In case of extrapulmonary involvement, the symptoms are pertaining to the organ involved.

We report a case study of disseminated tuberculosis in an immunocompetent patient where severe migratory joint pain without joint effusion was the dominating feature.

\section{Case Report}

A 26-year old male was admitted with a history of severe pain in his right hip, which he described to be insidious to begin with, gradually progressive in intensity, stabbing in nature and located closer to the midline. There was no history of radiation of this pain to either of the thighs. He gave a twomonth long history of multiple joint pains involving both small and large joints mainly of the left side and was migratory in nature. The small joints of left foot were involved first, followed by 
left hip, left shoulder, left elbow, left wrist and then the right foot was involved. There was no history of any prolonged fever, night sweats, weight loss, loss of appetite, cough or any other respiratory symptom. There was no history of any kind of trauma to the back. There was no history of lower back pain. There was no prolonged history of taking any drug. The patient denied having undergone any dental procedure or any history pertaining to throat or skin infections in his childhood. He was non-diabetic and normotensive.

On physical examination, he was afebrile and there was no remarkable finding apart from palpable supraclavicular and axillary lymph nodes, and tenderness over left sacroiliac region and left foot along fourth and fifth carpometacarpal joints. Rest of the involved joints were non-tender on presentation. No other lymph nodes were palpable.

Blood investigations showed a raised ESR (70mm in first hour), raised CRP (35.8 $\mathrm{mg} / \mathrm{L})$, raised alkaline phosphatase (222 U/L) and raised Serum ferritin levels (3404 ng/mL). Rest of the blood investigations came out to be within normal limits. Serology for Scrub Typhus, leptospirosis, EBV Tetrapanel, Malarial parasite, Widal, Typhidot IgM, Weil-Felix Test, NS1 Antigen, ANA came out negative. HLA-B27 was negative. ACE levels were within normal limits. Blood culture and urine culture were negative. Coagulation profile was normal. Montoux test was negative. HIV(I \& II), HBsAg, Anti-HCV were negative. Induced sputum was sent for $\mathrm{ZN}$ staining that came out to be negative. ASO titres were also raised (false positive).

X-rays were done for chest, left foot, right foot, left hip joint, left elbow and left wrist. All the Xrays came out clear except for right foot that showed lytic lesions at the base of second metatarsal and distal tibia.

USG-Abdomen and pelvis was done that showed splenomegaly along with multiple subcentimetric hypoechoic areas seen throughout splenic parenchyma. There were multiple enlarged peripancreatic and periportal lymph nodes. There was also evidence of hypoechoic nodules in prostate.

CECT-Chest and abdomen were done which showed multiple, enlarged, necrotic, conglomerate left paratracheal, subcarinal, axillary, azygoesophageal and left hilar lymph nodes. Liver was mildly enlarged in size. Spleen was enlarged and showed multiple rounded non-enhancing hypodense areas. There were multiple enlarged paraportal, peripancreatic and mesenteric lymphnodes.

CEMRI-Pelvis and Lumbosacral spine was done which showed multiple variable sized lesions involving sacrum, ilium and lumbar vertebrae. There was thickening of bilateral S1 nerve roots with post contrast enhancement. There was illdefined area in prostate on left side showing post contrast enhancement, likely prostatic abscess. There was thickened urinary bladder wall suggestive of cystitis. Possibilities of disseminated tuberculosis, sarcoidosis and fungal infection were included.

Whole Body FDG-PETCT was done which revealed hypermetabolic lesions involving bones (both axial and appendicular skeleton), spleen, right posterior and lateral pharyngeal wall and left paralaryngeal space, lymph nodes (supra and infradiaphragmatic) and prostate. Tubercular etiology was suspected and lymph node biopsy planned.

Left supraclavicular lymph node biopsy was done for confirmation which showed many epithelioid cell granulomas with Langhan's giant cells and areas of necrosis. ZN Stain for AFB was positive. A diagnosis of disseminated tuberculosis, involving bones, spleen, lymph nodes, pharynx and prostate, was made. Category I Antitubercular drugs were started along with analgesics for pain management. The patient is improving on ATT and is showing decrease in pain and regression of lymph nodes which were earlier palpable. 


\section{Osteoarticular Tuberculosis}

Osteoarticular tuberculosis is relatively rare extrapulmonary complication of Mycobacterium tuberculosis. 1-3\% of patients with TB present with skeletal involvement. ${ }^{1}$ Around half of these have spinal involvement and the rest have extraspinal osteoarticular joint involvement. ${ }^{2-}$ ${ }^{7}$ Monoarticular TB arthritis is usually more common from where the organism can be isolated from the joint. ${ }^{8}$ Osteoarticular TB occurs majorly by hematogenous spread from a primary focus like lung, kidney, lymph node, etc. or less commonly by direct spread from adjacent tissues. ${ }^{8-13}$ Direct invasion of synovium may also cause TB of joint, e.g. Poncet's arthritis. ${ }^{8-11}$ Non weight-bearing joint may also be involved such as wrist, elbow, and small joints of hands. These transmit via hematogenous route from visceral foci such as the lung, kidneys, lymph node or other viscera. ${ }^{14-18}$

Mild local and constitutional symptoms are more common that may lead to delays in diagnosis. The varied clinical presentation of the disease and sometimes lack of constitutional features contribute to the delay in the diagnosis of tubercular arthritis. ${ }^{6,19,20}$ This may result in additional bone or joint destruction, especially in patients with septic arthritis due to infection caused by mycobacterial species. ${ }^{6,21,22}$

Osteoarticular TB can mimic other disease due to its varied clinical presentation and radiographic appearance leading to long delay in diagnosis. ${ }^{23,24}$ There is also lack of awareness, insidious in onset, and often lack of constitutional or pulmonary involvement. Early diagnosis, specific and adequate treatment can be rewarding for maintaining good joint function by preserving the articular cartilage and joint space. Osteoarticular TB should be strongly suspected in the context of persistent monoarthritis in a susceptible host. The individuals who are imunocompromised, elderly or children in close contact to $\mathrm{TB}$ or those individuals who are under treatment with corticosteroid and/or immunosuppressive and biologic agent or those with history of trauma should undergo microbiological or histological tests for TB which remains the gold standard in the diagnosis of TB.

\section{Conclusion}

Tuberculosis is an old disease known for its varied clinical presentations. This disease is still a global medical emergency and should not be taken lightly by the clinicians, epidemiologists and microbiologists, more so in the emergence of HIV and diabetes. Tuberculosis should be considered in any patient with persistent pyrexia in highburden countries like India. Osteoarticular tuberculosis should always be kept among the differential diagnoses for the patients not responding to conventional analgesics and laboratory investigations are inconclusive for other diseases involving bones and joints. Delay of therapy should be avoided for want of definite microbiological diagnosis. Earlier administration of ATT in the appropriate dosage and for the recommended period reduces the morbidity and mortality of this global disease.

\section{References}

1. Malaviya AN, Kotwal PP (2003) Arthritis associated with tuberculosis. Best Pract Res ClinRheumatol 17: 319-343.

2. Iademarco MF, Castro KG (2003) Epidemiology of tuberculosis. Semin Respir Infect 18: 225-240.

3. Peto HM, Pratt RH, Harrington TA, LoBue PA, Armstrong LR (2009) Epidemiology of extrapulmonary tuberculosis in the United States, 1993-2006. Clin Infect Dis 49: 13501357.

4. Narang S (2012) Tuberculosis of the entheses. IntOrthop 36: 2373-2378.

5. Mateo L, Ruiz Manzano J, Olivé A, Manterola JM, Pérez R, et al. (2007) [Ostearticular tuberculosis. Study of 53 cases]. Med Clin (Barc) 129: 506-509.

6. Samuel S, Boopalan PR, Alexander M, Ismavel R, Varghese VD, et al. (2011) 
Tuberculosis of and around the ankle. J Foot Ankle Surg 50: 466-472.

7. Harisinghani MG, McLoud TC, Shepard JA, Ko JP, Shroff MM, et al. (2000) Tuberculosis from head to toe. Radiographics 20: 449-470.

8. Abdulaziz S, Almoallim $\mathrm{H}$, Ibrahim A, Samannodi M, Shabrawishi M, et al. (2012) Poncet's disease (reactive arthritis associated with tuberculosis): retrospective case series and review of literature. Clin Rheumatol 31: 1521-1528.

9. Abdelwahab IF, Bianchi S, Martinoli C, Klein M, Hermann G (2006) Atypical extraspinal musculoskeletal tuberculosis in immunocompetent patients: part II, tuberculous myositis, tuberculous bursitis, and tuberculoustenosynovites. Can AssocRadiol J: 278-286.

10. Poncet A (1897) De la polyarthritestuberculeusedeformanteoupseudo rheumatismechroniquetuberculeux.

CongresFrancaise de chirurgerie 1: 732.

11. Netval M, Hudec T, Hach J (2007) [Our experience with total knee arthroplasty following tuberculous arthritis (1980-2005)]. ActaChirOrthopTraumatolCech 74: 111-113.

12. Sequeira W, Co H, Block JA (2000) Osteoarticular tuberculosis: current diagnosis and treatment. Am J Ther 7: 393-398.

13. Chenskï̈ $\neg$ EP, Omirova KT, Sule $\ddot{A} \neg$ manovBSh (1980) [Characteristics of osteoarticular tuberculosis patient contingents in Kazakhstan and the ways for their detection]. ProblTuberk 6-8.

14. Tuli SM (2002) General principles of osteoarticular tuberculosis. ClinOrthopRelat Res: 11-19.

15. Leibe H, Köhler H, Kessler P (1982) [Osteoarticular tuberculosis. Review - current status of diagnosis and therapy]. ZentralblChir 107: 322-342.
16. Magnussen A, Dinneen A, Ramesh P (2013) Osteoarticular tuberculosis: increasing incidence of a difficult clinical diagnosis. $\mathrm{Br} \mathrm{J}$ Gen Pract 63: 385-386.

17. Scanzello CR, Goldring SR (2012) The role of synovitis in osteoarthritis pathogenesis. Bone 51: 249-257.

18. de Lange-Brokaar BJ, Ioan-Facsinay A, Yusuf E, Visser AW, Kroon HM, et al. (2013) Degree of synovitis on MRI by comprehensive whole knee semi-quantitative scoring method correlates with histologic and macroscopic features of synovial tissue inflammation in knee osteoarthritis. Osteoarthritis Cartilage

19. Walker GF (1968) Failure of early recognition of skeletal tuberculosis. Br Med J 1: 682-683.

20. Carli P, Landais C, Aletti M, Cournac JM, Poisnel E, et al. (2009) [Current treatment of rheumatoid arthritis]. Rev Med Interne 30: 1067-1079.

21. Hsiao CH, Cheng A, Huang YT, Liao CH, Hsueh PR (2013) Clinical and pathological characteristics of mycobacterial tenosynovitis and arthritis. Infection 41: 457-464.

22. Foocharoen C, Nanagara R, Foocharoen T, Mootsikapun P, Suwannaroj S, et al. (2010) Clinical features of tuberculous septic arthritis in KhonKaen, Thailand: a 10-year retrospective study. Southeast Asian J Trop Med Public Health 41: 1438-1446.

23. Ellis ME, el-Ramahi KM, al-Dalaan AN (1993) Tuberculosis of peripheral joints: a dilemma in diagnosis. Tuber Lung Dis 74: 399-404.

24. Tsuduki E, Kawada H, Takeda Y, Toyoda E, Kobayashi N, et al. (2002) [A case of multiple bone and joint tuberculosis which had been misdiagnosed as the rheumatoid arthritis and treated with prednisolone for eleven months]. Kekkaku 77: 361-366. 